Sādhanā Vol. 39, Part 1, February 2014, pp. 97-121. (C) Indian Academy of Sciences

\title{
Automatic Tamil lyric generation based on ontological interpretation for semantics
}

\author{
RAJESWARI SRIDHAR*, D JALIN GLADIS, \\ KAMESWARAN GANGA and G DHIVYA PRABHA
}

Department of Computer Science and Engineering, Anna University, Chennai 600 025, India

e-mail: rajisridhar@gmail.com; jalingladis18@gmail.com; kss.ganga@gmail.com; be.cooldivi@gmail.com

MS received 19 June 2012; revised 22 August 2013; accepted 22 August 2013

\begin{abstract}
This system proposes an $N$-gram based approach to automatic Tamil lyric generation, by the ontological semantic interpretation of the input scene. The approach is based on identifying the semantics conveyed in the scenario, thereby making the system understand the situation and generate lyrics accordingly. The heart of the system includes the ontological interpretation of the scenario, and the selection of the appropriate tri-grams for generating the lyrics. To fulfill this, we have designed a new ontology with weighted edges, where the edges correspond to a set of sentences, which indicate a relationship, and are represented as a tri-gram. Once the appropriate tri-grams are selected, the root words from these tri-grams are sent to the morphological generator, to form words in their packed form. These words are then assembled to form the final lyrics. Parameters of poetry like rhyme, alliteration, simile, vocative words, etc., are also taken care of by the system. Using this approach, we achieved an average accuracy of $77.3 \%$ with respect to the exact semantic details being conveyed in the generated lyrics.
\end{abstract}

Keywords. Tamil lyric; lyric generation; ontology; semantics; $N$-gram.

\section{Introduction}

Natural language generation is gaining popularity by means of research in the Automatic Question and Answer generation system (Hirschman \& Gaizauskas 2002), Automatic text mining (Apte \& Weiss 1997), Automatic Sentence Analysis and Generation (Mann \& Moore 1981), etc. In this context, a new domain which is gaining popularity is the automatic poetry generation system (Wong 2008). A variant of poetry is termed 'lyric' (Bowra 1961), which is poetry written for movies. In this work, we have attempted an automatic lyric generation system.

*For correspondence 
Human Lyric generation can be attempted using two broad approaches, namely, tune-based and context-based (Suriyah et al 2011). Tune-based generation is one in which the lyrics are written according to a given tune, which is specified either as a 'Sandam', or as a piece of tune using the keyboard. The lyrics generated in this manner are supposed to collectively convey a mood, and ready to be sung. The other approach, namely, context-based approach is one, where the situation is given as input in a textual form; this information is analysed and the words are chosen to generate one or more lyrics appropriately to portray the scenario. A tune is set to the generated lyrics and the lyrics are sung to this tune. The problem with the tune-based approach is that the semantics and the context of lyrics are compromised, since the tune is given importance. On the other hand, the problem with the context-based approach is that the lyrics are given importance and some words in the lyrics need to be revised to fit the tune. In this work, we have developed an automatic lyric generation system for the Tamil language, which uses the context-based strategy. The context-based approach was chosen to convey the emotion of the given situation. In this work, we use an ontology to determine the semantic information from the scene, and use it to generate lyrics using Tamil sentence generation rules. Generating narrative, vivid and picturesque lyrics is the aim of this system.

The paper is organized as follows: Section 2 discusses the previous work on lyric generation. Section 3 deals with the system overview and the various modules in the system architecture. Section 4 focuses on the results and section 5 gives the conclusion along with the future work, and extensions to the system.

\section{Literature survey}

One of the existing works on lyric generation for the Tamil language concentrates on generating lyrics from a given melody (Sobha \& Ananth Ramakrishnan 2010). This is a tune-based lyric generating system wherein the input to the system is a tune in the KNM representation, where ' $\mathrm{K}$ ' refers to 'Kuril', ' $\mathrm{N}$ ' means 'Nedil' and ' $\mathrm{M}$ ' means 'Muttru' and this is called the 'asai' pattern in Tamil. The core of this system lies in choosing a selection restriction rule. A selection restriction rule is one which has a verb along with the characteristics of the subject that can occur, viz., the object that is generally used with it, and any other specific characteristics describing the subject and the object. An example of a selection restriction rule is $\mathrm{pAr}=>[+$ living, + animate, + vertebrate, + mammal, + human $] ; \mathrm{NOM}=>$ [no_obj]. Once such a rule is selected, the next step is to identify the actual subject and object, which are to be used in the resulting lyrics. This selection of subject and object is done based on the hints obtained from the selection restriction rule. Once the subject and object are chosen, they are coined to frame a proper sentence conveying a meaning. At this stage, the line is matched against the given 'asai' pattern, to check if it suits the given input tune. If so, the process continues for generating the other lines for the lyrics. If it does not match, this generated line is discarded, and a new selection restriction rule is selected, and the process continues until a line that matches the given input tune is obtained. In another work of Ananth Ramakrishnan et al (2009), the authors attempted to generate Tamil lyrics for a given tune by training the system with a corpus of 10 melodies to understand the syllable pattern. The $\mathrm{CRF}++$ tool kit is used for learning the lyric-syllable pattern. Having these trained models as samples, the syllables of new melodies are produced, resulting in the generation of lyrics. As both their systems are tune-based approaches, their systems had restrictions in the choice of words, and not conveying the essence of the scenario.

Hence, to study the context-based lyric generation process, we referred to yet another work of Hugo Goncalo Oliveira, 'Portugal, PoeTryMe' (Oliveira 2009). PoeTryMe is a poetry generation 
platform under development, which intends to help the automatic generation of meaningful poetry in the Portuguese language, according to a given semantics. To get it done, the authors have constructed a semantic network, which is processed to aid in lyric generation. The generation of a sentence/verse starts with a set of key terms, which are used to select a sub-graph from the main semantic graph. All the relations that cannot be mapped to rules in the grammar are removed, and all the relations involving the given terms are included in the sub-graph. After selecting a random term from the sub-graph, all the relations that are involved are obtained. One of those relations is then randomly selected and mapped to a set of generation rules included in the grammar. The authors have attempted three different strategies for lyric generation, namely, 'basic', 'generate' and 'test and evolutionary'. The final lyric is generated by choosing sentences from one or a combination of their strategies. This paper gives an idea of a possible technique to extract the semantic details from a scenario, but the generation strategy needs to be designed for the Tamil language.

In order to generate Tamil sentences, we referred to the work of Pandian \& Geetha (2009) to get rules on sentence generation strategies. The authors have proposed a machine learning technique for Tamil sentence generation. The input to the system is a set of concept terms and semantic roles for which the system generates simple Tamil sentences using tri-gram models. The authors speak about a module called 'the learner', which is able to realize the semantics and generate a meaningful sentence based on it, realizing the concept conveyed. The learner does Part-of-Speech (POS) Tagging, Chunking and Semantic role labelling. The collective results of these were used in developing a bi-gram model. The bi-gram model gives the best choice of words that would make a meaningful sentence. These works gave us a grasp of the actual lyric generation process.

In addition, to get an idea on how the lyricists make use of words and rhyming strategies, we referred to the lyric analysis work of Karthika et al (2011). In this work, the authors propose a data analysis model for words, rhymes and their usage in Tamil lyrics. This paper talks about analysis of lyrics in three different ways, namely, 'word analysis', 'rhyme analysis', and 'cooccurrence concept analysis', for which the authors have used an algorithm called the apriori algorithm. The apriori algorithm is an influential algorithm which uses a 'bottom up' approach, where frequent subsets are extended one item at a time (a step known as candidate generation), and groups of candidates are tested against the data. The algorithm terminates when no further successful extensions are found. The rhyme analysis checks for 'Edugai' (Alliteration), 'Monai' (Rhyme), and 'Eyaibu' (End rhyme). This paper highlighted to us the need for rhyme in lyrics, which was incorporated in the generated lyrics to enrich their poetic characteristics. In another work, Suriyah et al (2011) aimed at building special indices for the lyric generator framework of Karthika et al (2011). The authors have used POS and rhyme features, to retrieve words from a corpus for generating lyrics. The indexing is based on the KNM (Kuril, Nedil, Mei) pattern, and the letters that occur in the rhyming spots of the words. The authors have constructed separate hash tables for each POS and rhyming scheme to aid faster retrieval. This work is also aimed at generating lyrics based on tune.

The works discussed so far have focused on generating lyrics using the tune, which has limitations, as already discussed. Therefore, the goal of this paper is to design a Tamil lyric generation system, by incorporating semantic information, sentence generation strategies, and rhyming words, all of which are discussed in the following section.

\section{System overview}

The system aims at generating meaningful lyrics for a given situation. Making the system understand the given scenario is what we deliberate on. Initially, we started our work by identifying 
the emotion of the scenario and generated lyrics pertaining to the emotion based on a general tri-gram model (Sridhar et al 2012b). However, as mentioned in that work, the lyrics generated by the system pertained to the emotion, but did not convey the complete context of the input scenario. In addition, the adjacent lines of the lyrics were not correlated. But correlation between successive lines and conveyance of the input situation were our goals; hence, we tried to create a graphical model to understand the given scenario, for which we referred to the work done by Oliveira (2009), who has constructed a semantic network. But, for our work, we concluded that we required relationship among the entities. Therefore, we constructed an ontology, as against the semantic network, to interpret the given scenario, where we proposed a new idea of including information in the edges of the ontology. The information is included as a tri-gram model indicating that the entities are related using a relationship that is described in one or more sentences represented as a combination of words.

The input to the system is the vernacular description of the scene. The process begins with the removal of stop words, followed by the ontological interpretation, which extracts the semantics of the input scene. The semantics thus obtained guide us to the domain from where the words have to be gathered to convey the exact story given in the scene. While selecting the words, rhyming is taken care of to avoid excessive post-processing work. These root words are used by the morphological generator to frame the lyrics, which form the output after the addition of singer information and vocative words.

We have made contributions in the following blocks of the system architecture, given in figure 1 .

- The scrutinizer-Designed for stop words removal, based on a look up list approach.

- Morphological analyzer-Modified the analyzer from TACola (Tamil Computing Lab), to incorporate the positive and negative senses conveyed in the root word.

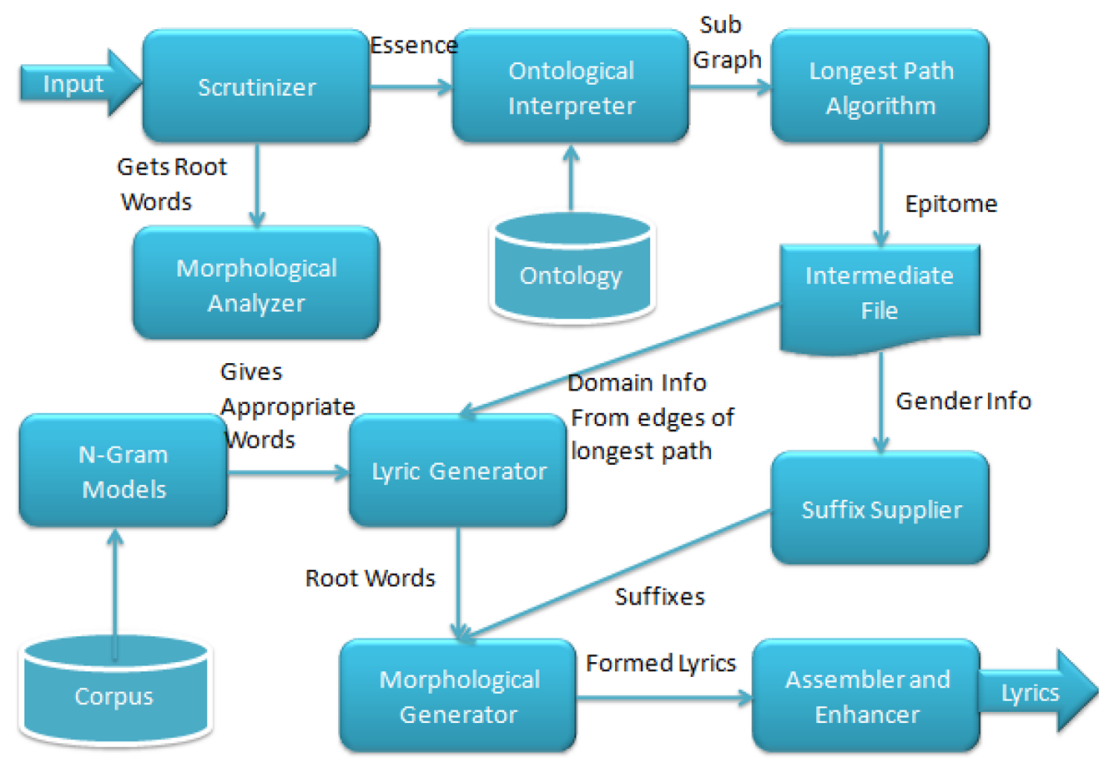

Figure 1. System architecture of the lyric generation system. 
- Ontology with weighted edges-New algorithm has been designed, where the nodes in the network are words that are commonly used in describing the input scenario, and the edges represent a relationship between these nodes that corresponds to a set of sentences represented as a tri-gram.

- Ontological interpreter-Used for extracting the epitome of the scenario from the designed ontology.

- Lyric generator-Picks words from the tri-grams and assembles them to form the lyrics, by ensuring the presence of rhyming information.

- $N$-gram modules-A randomized approach for selecting root words from the designed bigrams and tri-grams of words separated into various domains which are referred to by the lyric generator for generating the final lyrics. This is different from the maximum probabilistic path typically used in a bi-gram decoder for the choice of words.

- Suffix supplier-Module that supplies the suffix information needed to coin the words to their packed form, which includes the tense, gender and 'urubu' information.

- Morphological generator-Designed to use the information provided by the suffix supplier to coin the words to their complete forms. It analyses the paradigms of the words to coin them to their correct packed forms.

- Assembler and enhancer module-Designed to add singer information and vocative words to the generated lyrics to make them more pragmatic.

The following section gives a brief description of the modules of our proposed system.

\subsection{Scrutinizer}

The scrutinizer receives the vernacular description of the input scene from the user. The process starts with the removal of stop words from the scene, thereby reducing the word set that has to be processed. This is done by referring to the available list of stop words. Then the scrutinizer identifies the actions involved in the given scene and extracts the gender doing those actions from the scene. This is useful in adding vocative words and at the morphological generation stages of lyric generation. Hence, this information is written in the intermediate file. Then it calls for the morphological analyzer to get the root words for all the words available in the scene.

\subsection{Morphological analyzer}

The Morphological analyzer takes the scenario as input, in which the stop words are removed. It takes each word and identifies the root word for each of these and writes them back to a file which will be used by the ontological interpreter. However, we have to modify the analyzer to extract and retain more information from the input scene. For example, when identifying the root words, care should be taken that the actual meaning conveyed in the input word is not truncated. For example, the Tamil words 'sandhiththanar' (meaning 'met') and 'sandhikkaamal' (meaning 'did not meet') will be truncated by the analyzer, to the root word 'sandhi' which means 'meet'. In such cases, the positive and negative sense conveyed in the actual word in the input gets hidden in the root word. So the morphological analyzer obtained from 'TaCoLa, Anna University' was modified to handle this by observing the root word along with its suffix. This additional information was also included in the intermediate file to ensure that the meaning of the input scenario is conserved for subsequent processing. 


\subsection{Ontology with weighted edges}

The core of the lyric generation system is the ontology that was used to handle the semantics of the entire system. In this work, we have modified the ontology as defined in the literature to suit our need by adding weights to the edges. Typically, ontology defines a common vocabulary for researchers who need to share information in a domain (Spyns et al 2002). It includes machineinterpretable definitions of basic concepts in the domain, and the relations among them. Ontology has been used to share the common understanding of the structure of information among people or software agents, to enable the reuse of domain knowledge, to make domain assumptions explicit, to separate domain knowledge from the operational knowledge, and to analyse domain knowledge. In all these cases, the edges in the ontology have simple descriptions of the relationship between the nodes (Mahalingam \& Huhns 1998). In our system, the ontology is modified to have tri-grams at the edges, thereby indicating a need to represent the relationship among entities as a sequence of words.

The interpretation of the scenario given is the heart of the system, and to get it done, we have chosen the technique of building an ontology with the words that are commonly used in describing the given situation. The edges in the ontology represent a relationship between the nodes in the ontological network. But this relationship is not just represented by a single 'word' or a 'paradigm'. In our case, we use tri-grams at the edges to bring out this relationship, to get a better domain knowledge, and hence, we call this as 'ontology with weighted edges'. The ontology is a semantic network which is represented in the form of an ' $N$ ' $\mathrm{x}$ ' $\mathrm{N}$ ' adjacency matrix. The nodes $\mathrm{N}_{1}, \mathrm{~N}_{2}, \ldots, \mathrm{N}_{n}$ are the words used in the scene description and each of the edges $\mathrm{E}_{12}, \mathrm{E}_{13} \ldots \mathrm{E}_{i j}$ correspond to the tri-grams built on it, which consist of words confined to the context of that edge. An edge $\mathrm{E}_{i j}$ exists from a node $\mathrm{N}_{i}$ to a node $\mathrm{N}_{j}$, only if the word

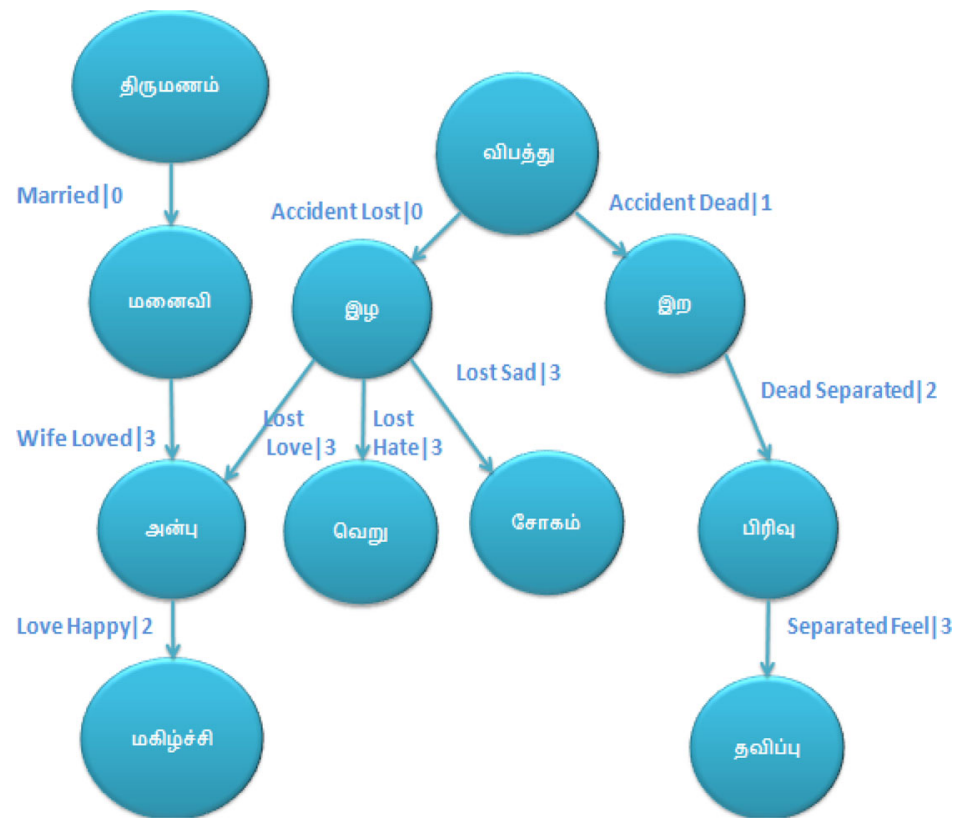

Figure 2. Part of ontology built for the lyric generation process. 
corresponding to the node $\mathrm{N}_{i}$ when followed by the word corresponding to the node $\mathrm{N}_{j}$ conveys sense. There are also edge weights $\mathrm{W}_{i j}$ 's for each edge $\mathrm{E}_{i j}$ to depict the significance of that link, in understanding the semantics of the scenario. This ontology with weighted edges is built by analysing the various domains and gathering a set of words, which are used to describe the various scenarios in those domains. With the collected words as nodes we constructed a semantic network, by finding the association between all the nodes. After the semantic network is constructed, we placed tri-grams on the edges of the network to define the relationship between its corresponding nodes, and also edge weights to determine the number of lines to be generated from that tri-gram. An example of our ontology with weighted edges is shown in figure 2 .

This part of the entire ontology that is displayed in figure 2, is built to handle scenarios where somebody meets with an accident, and the nodes here show the consequences of the accident. The sub graph corresponding to these has words 'Thirumanam', 'Manaivi', 'Vibathu', 'Ira', 'Izha' etc., that are commonly used in describing the scenario, and these nodes are connected via weighted edges which have tri-grams in them to convey the semantics. For example, considering the link connecting the nodes 'Vibathu' $\rightarrow$ 'Ira', the link here has a tri-gram in it 'Accident Dead' and this link helps in understanding that a person has died in an accident. The edge weight ' 1 ' indicates the significance of this link in understanding the semantics and also contributes to the number of lines in the final lyrics. If the edge weight is ' 0 ' it has no special significance in the resulting lyrics, but it needs to understand the scenario in its finer details.

\subsection{Ontological interpreter}

The ontological interpreter first gets the epitome of the scene. This is done by finding the set of words that occurs both in the input scene and the ontology. The epitome thus extracted is

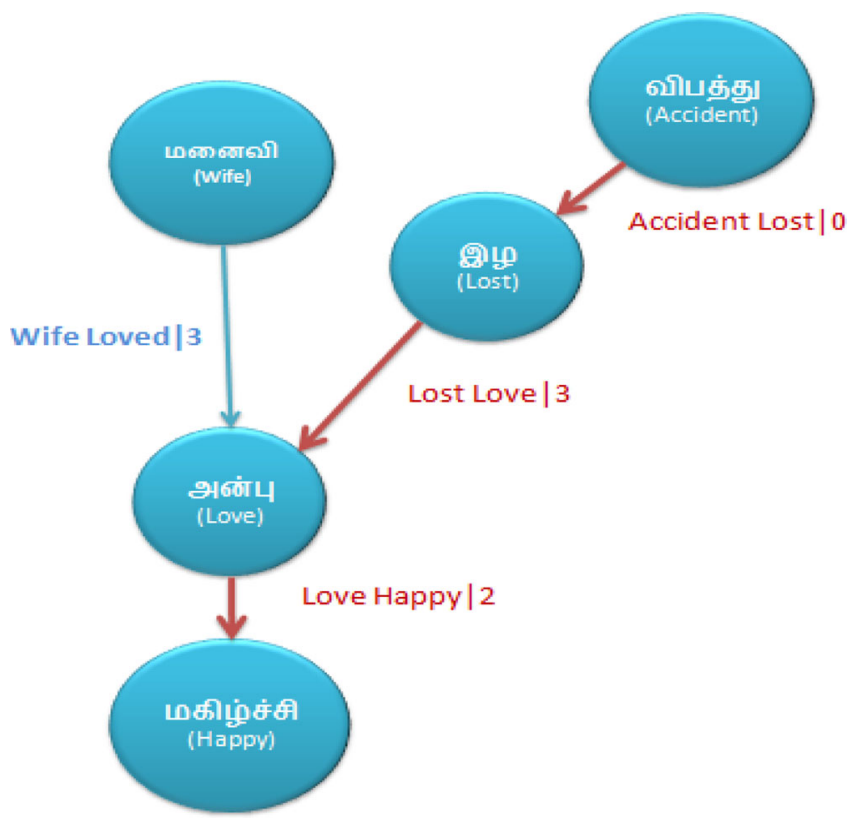

Figure 3. Sub-graph extracted from the entire ontology. 


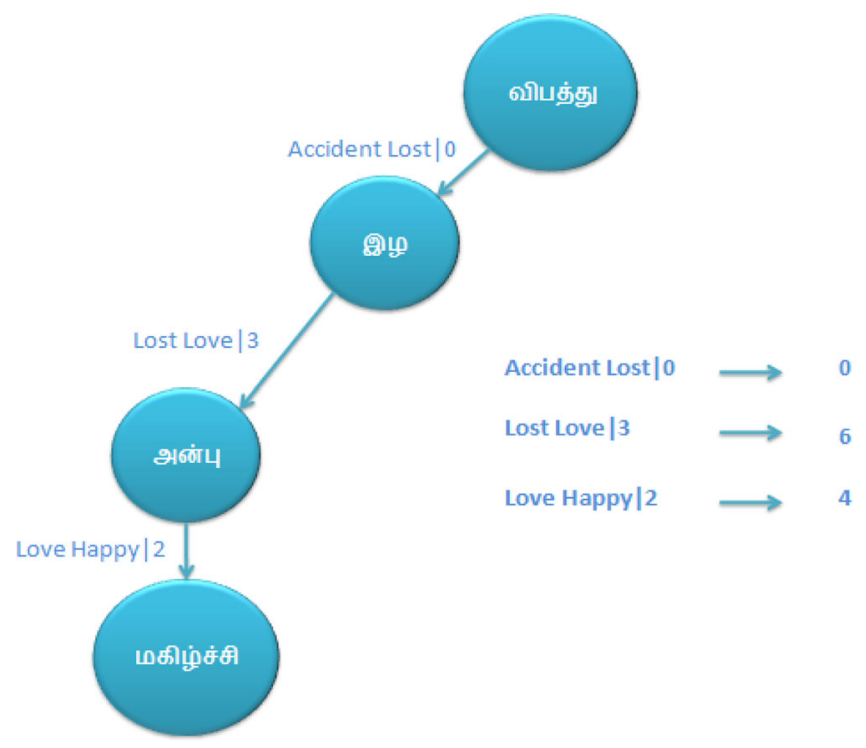

Figure 4. Longest path determined by the backtracking algorithm.

the narrowed set of words, which will contribute to the actual lyric generation process. With the words of the epitome as nodes, a sub-graph pertaining to this scenario is extracted from the entire ontological network by observing the tri-gram edges. This sub-graph of the actual network is then processed to make the system understand the scenario. The adjacency matrix of the subgraph is written in the intermediate file for future use. The sub-graph extracted for an input scene below is displayed in figure 3 .

The highlighted words in the given scenario (and the corresponding transliteration is given below) were found in the ontology, and the sub-graph corresponding to this part is extracted from the entire ontological network. The translation of the scenario is 'One person looses his leg in an accident. His wife is very supportive to him. He is happy of his wife's love and care'. For this scenario in Tamil, the sub-graph is shown in figure 3. This is represented as an adjacency matrix, and is processed to get the longest path which is shown in figure 4. This longest path which has nodes 'Vibathu' $\rightarrow$ 'Izha' $\rightarrow$ 'Anbu' $\rightarrow$ 'Magizhchi' helps the system understand the details, that the person is physically impaired, and his life partner loves him a lot even after the mishap and they lead a happy life.

அவன் ஒரு விபத்தில் தன் கால்களை இழக்கிறான் . அவன் மனைவி

அவனுக்கு அன்பாகவும் ஆறுதலாகவும் இருக்கிறாள் . அவன் தன்

மனைவி தன் மேல் வைத்திருந்த காதலை எண்ணி மகிழ்ச்சி

அடைகிறான் .

Avan oru vibathil than kaalgalai izhakkiraan. Avan manaivi Avanukku anbaagavum aaruthalaagavum irukkiraal. Avan than Manaivi than mel veithirundha kaadhalai enni magizhchi Adaikiraan. 


\subsection{Longest path algorithm}

The sub-graph consists of many paths, which have words with equal or more emphasis. In order to ensure that the sub-graph enhances the semantic information of the lyrics to be generated, we decided to determine the longest path in the sub-graph, and use the words comprising that path for lyric generation.

The longest path problem belongs to the NP class (Thomas et al 2008), which means that the optimal solution cannot be found in polynomial time unless $\mathrm{P}=$ NP. We have used a back tracking algorithm (Weiss 2006) to determine the longest path, and hence, we compute the lengths of all the paths in the given graph, to arrive at an optimal solution. The longest path (LP) is chosen by the following relationship given in Eq. (1).

$$
\begin{gathered}
\mathrm{P}(\mathrm{u}, \mathrm{v})=\text { Simple path from } \mathrm{u} \text { to } \mathrm{v} \\
|\mathrm{P}(\mathrm{u}, \mathrm{v})|=\text { The number of edges in } \mathrm{P}(\mathrm{u}, \mathrm{v}) \\
L P(x, y)=\max _{x, y \in V}|P(x, y)| .
\end{gathered}
$$

The longest path thus obtained, will also be a sub-graph of the existing ontology, and so each of the edges along the path has a tri-gram and an edge weight associated with it, which is referred to during the lyric generation process. The longest path determined for the sub-graph shown in figure 3 is exhibited in figure 4.

\section{$3.6 \mathrm{~N}$-gram models}

In this work, we use a combination of the tri-gram and bi-gram models to choose the appropriate words for the sentences of the output lyrics. We choose every word in a sentence from the trigram with reference to the previous two words. In that case, the first word is chosen randomly, and second word is chosen using a bi-gram. This is done to achieve better coherence between the first two words. The tri-grams and bi-grams contain root words that are used to form the lyrics. These are placed on the edges of the ontological network. A tri-gram model located on an edge $\mathrm{E}_{i j}$ will contain only the words confined to the context conveyed in the edge $\mathrm{E}_{i j}$. Thus, any sentence framed from that tri-gram will convey the semantics related to that context. Bigrams are used here, to get the first two words of any line in the lyrics. The first word is chosen randomly from the bi-gram and the word following it is chosen, based on a probabilistic measure from the bi-gram, so that there is a good choice of words for the lyrics. Typically, the choice of words from a bi-gram will be determined by a decoder such that the words along the path yield a maximum probabilistic value. However, this algorithm, if incorporated for choosing the words for a lyric, will always yield the same set of words. In order to avoid this, and make the lyric generation process more creative, we converted this choice of words as a randomized probabilistic measure, from a list of probabilistic values for the edge length.

\subsection{Lyric generator}

The actual lyric generation process occurs in two phases. To give a detailed note, the odd lines in the lyrics are generated by picking a random initial word and consulting the tri-gram of the 
ontology edge to get the succeeding words, thereby forming one line of lyric which is explained in a greater depth in section 3.7a. The even lines in the lyrics are generated giving importance to the end rhyme with respect to their immediate odd lines. This relationship between adjacent lines ensures that there is coherence between them. This phase is explained in detail in section $3.7 \mathrm{~b}$.

3.7a The lyric model: The generation of every odd numbered line in the lyrics makes use of this strategy. The lyric model is based on semantic interpretation. To bring this semantic correctness, we propose a tri-gram model where the words are chosen relative to the previous ones. For better performance, we adopt a technique that makes use of a blend of words and co-occurrences. The tri-gram is constructed based on a probabilistic measure to ensure that the most suited words form a sentence and the correlation is maintained. A randomization technique on the words to be selected is used to ensure that the same words are not chosen always for similar situations. Thus, this is a randomized probabilistic tri-gram model. Once when we have identified the longest path, we go to the corresponding tri-gram model available in each edge $\mathrm{E}_{i j}$, and choose the words to form the lyrics. Initially, a seed word say $\mathrm{S}_{1}$ is chosen in a random manner, and using the bi-gram model designed, we go for selecting the next word $\mathrm{S}_{2 i}$, based on the probability of the occurrence of that particular word $S_{2 i}$ following the previous word $S_{1}$, and let this probability be $\mathrm{P}_{2 i}$. We repeat the process of selecting $\mathrm{S}_{2}$ for this $\mathrm{S}_{1}$, and hence, we will have a set of $S_{2 i}$ 's with the respective probabilities $P_{2 i}$ 's. After selecting the first two words, the remaining words are chosen based on the tri-gram for each of the previously occurring word pair $<\mathrm{S}_{1} ; \mathrm{S}_{2}>$.

After determining the probable sequence of words based on a defined first word, which is specified by $<\mathrm{S}_{1} ; \mathrm{S}_{2 i} ; \mathrm{S}_{3 i}>$, for all ' $i$ ' in the tri-gram, we compute a cumulative probability for these sets, using Baye's theorem. Out of these the triple $<\mathrm{S}_{1} ; \mathrm{S}_{2 i} ; \mathrm{S}_{3 i}>$ that yields the maximum probability for any ' $i$ ', is selected for further processing. The words obtained are the root words, and hence, they are sent to the morphological generator. The process continues for the required number of times, based on the edge weights.

3.7b Rhyming for coherence: The generation of every odd sentence is as described above, but the even sentences are generated considering the ending word (eyaibu) of the previous sentence. To elaborate, after the odd line of lyric gets generated, the end word (say S) is saved, and the probable set of words that could contribute to the end word of the even line in a lyric, is extracted from the tri-grams. The appropriate end word of this even line is chosen in an iterative fashion by referring to the suffix of the end word $\mathrm{W}$ of the odd line, that gets attached by the suffix supplier and the morphological generator (explained later).

After determining the suffix based on the odd line, the set of words that would constitute the last word is coined, using the suffix of the last word of the odd line. At this point, we have a probable set of words that matches the suffix of the last word of the odd line. These words (say $\mathrm{S}_{1}, \mathrm{~S}_{2}, \mathrm{~S}_{3}, \ldots, \mathrm{S}_{n}$ ) are stored in their packed forms and compared with the word $\mathrm{W}$, to check if it has the longest suffix match in terms of the end rhyme. The one that gives the longest match is chosen as a seed word to generate the even line. This seed word obtained will be the last word in that line, and the other words in the line are chosen in a reverse manner by referring to the tri-grams. But there are also chances that the word that occurred in the immediate previous sentence may get selected during the longest match calculation. Hence, care is taken to see that the words that are in the previous lines, are not chosen for the successive ones. 
The procedure explained so far is depicted in the following pseudo-code:

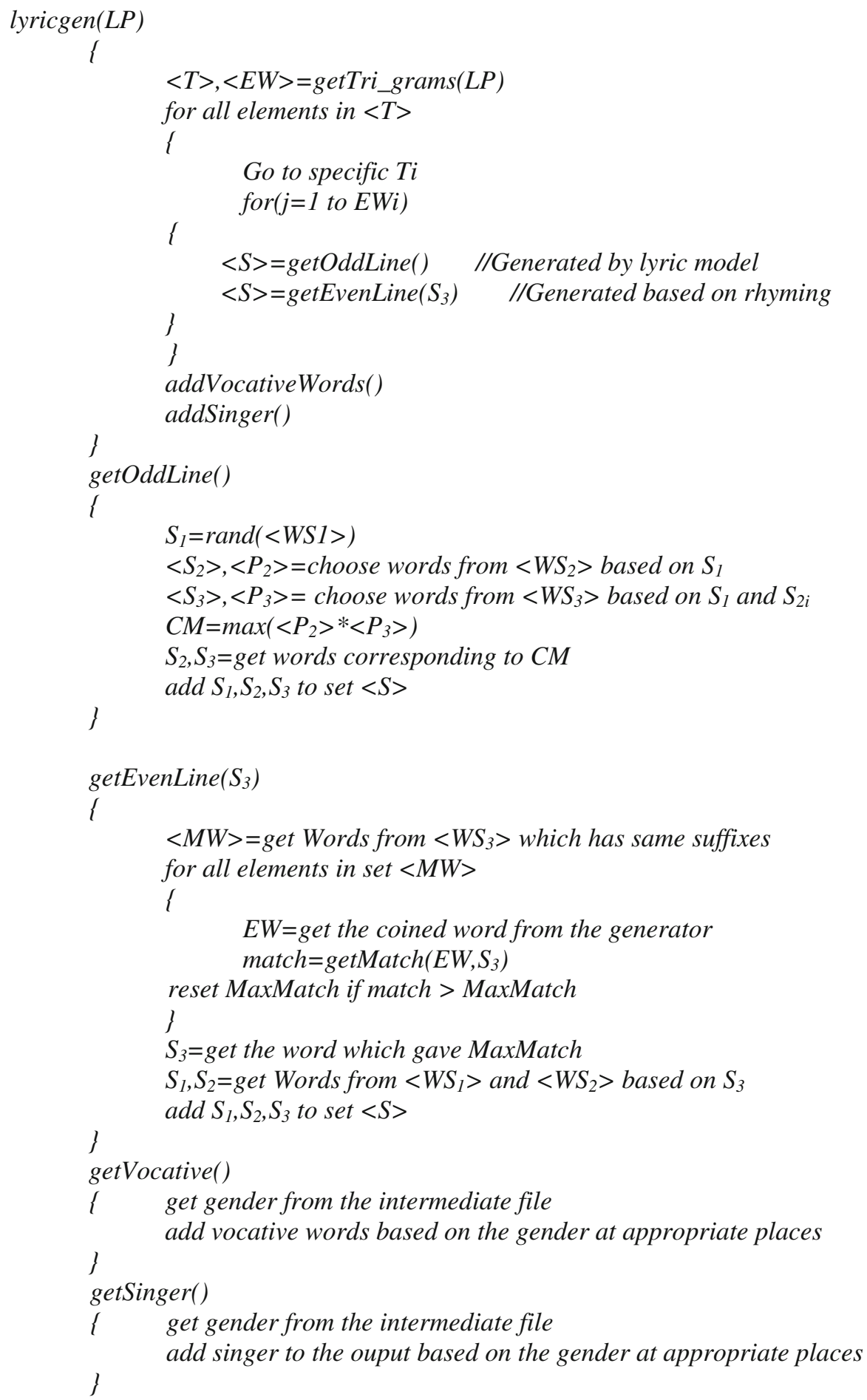




\subsection{Suffix supplier}

The suffix supplier provides the suffix for coining the words obtained from the lyric generator into their packed form. We have designed the suffix supplier by referring to the grammar rules of Tamil language. This module collects gender details from the intermediate file and chooses the appropriate 'urubu'. Hence, the details that it gives to the morphological generator are the tense, gender, rhyme and 'urubu' information. An example of the suffix information provided by the suffix supplier for the words that form a tri-gram 'Kaadhal'| 'Vizhu'| 'Un' is 'il|PAT, $1^{\text {st }} \mid$ aal'. In the suffix information shown in the example 'il|PAT, $1^{\text {st }} \mid$ aal', PAT refers to 'Past' tense and ' 1 st' refers to first person. When this is given as an input to the morphological generator, it coins the words to their packed form as 'Kaadhalil vizhundhaen unnal'.

\subsection{Morphological generator}

We have designed a morphological generator for our work to generate words, given the root, urubu, gender and rhyming information. We have referred to Tamil grammar rules for combining words, based on omitting or adding or replacing characters to join the root with the suffix. This module receives the root words from the lyric generator module. It initially identifies whether it is a noun or verb, to choose the appropriate technique of generation. Then, it examines the nature and ending of the root word to decide on the paradigm to which the word belongs. After the paradigm is identified, it utilizes the suitable Tamil grammar rules to find out the form of suffix, which has to be appended, and using that suffix it makes up the word.

\subsection{Assembler and enhancer}

This is the module that finally gathers all the generated sentences and if the number of lines generated are less than the threshold set (in our case, it is 8 , which can be modified), it generates the remaining number of lines for the last emotion that was conveyed, so that the threshold is met. This module also marks the perspective of the singers (singer identification). This is done by making use of the information collected from the scrutinizer. The scrutinizer identifies the actions and the genders performing those actions, and based on this, we learn who can sing a particular line in the lyrics. We use this information to mark the singers for the set of lines, as to whether a sequence of lines could be sung by a male or female. The same information is also used to add vocative words (words like 'anbae', 'sagiyae', 'kannae', which are some means of conveying 'love') to the lines generated, to make the generated lyrics resemble human composition.

\section{Result analysis}

The proposed system is generic for any domain. As of now, we concentrated on a few domains like love, betrayal, friendship and sadness. Initially, we built a corpus of about 2000 distinct Tamil root words, collected from about 1000 Tamil songs in these domains. Then, we computed the probability of the words, and constructed the various tri-gram models. The ontology was constructed with 100 different nodes that would help in a semantic interpretation. The tri-grams constructed with the words were aligned to the various edges of the ontology. The system would generate a minimum of 8 lines for each song, and the maximum is between 10 and 15. 
Then we increased the corpus to 10,000 distinct root words by referring to 3000 Tamil songs and analysed the response of the system. The system proved to be less responsive to the number of words in the corpus. We also checked the EMILLE corpus. The EMILLE corpus needed to be scrubbed and cleaned, to make it become part of the tri-gram model. We analysed the word sequence in the EMILLE corpus, which was for text processing, and hence the tri-gram/bi-gram probability values differed from the values we had in our system. Hence, we decided to add root words from the EMILLE corpus and retained our previously computed tri-gram and bi-gram probability values. The system was tested for 100 different scenes, comprising different domains of love. The following sub-domains of love were handled:

$$
\begin{aligned}
& \text { Love - Hate/betrayal } \\
& \text { Love - Happy/sad } \\
& \text { Love - Friendship } \\
& \text { Love - Relationship }
\end{aligned}
$$

Each module in the system is tested separately, and the stage-by-stage testing of the system is discussed in the next section.

\subsection{Output of various modules}

4.1a Input scene: The input scene and its transliteration are shown in figure 5 was given to the system and the results obtained are listed in the following subsections.

The meaning of the above input scene is:

"A boy and a girl loved each other through telephone conversation without seeing each other. The man attempts to visualize his lover's beauty on the previous night of their scheduled meeting day".

4.1b After scrutinization: The scrutinizer produces the essence from the scene (figure 6) and also finds the actions involved, and the genders from the scene (figure 7). The figures 6 and 7 also gives their transliteration for ease of understanding for non-Tamil speakers.

The input scenario here is analysed to remove the stop words, like 'avan', 'aval', 'than', etc., based on a look up list approach. After the stop words removal process, the actions involved in the scene, and the gender performing those actions are identified. The gender for a particular action can be found by analysing the suffix of that word or the words following it. Tamil grammar was referred to, to create rules to extract this information. In this example, 'azhagu' does not

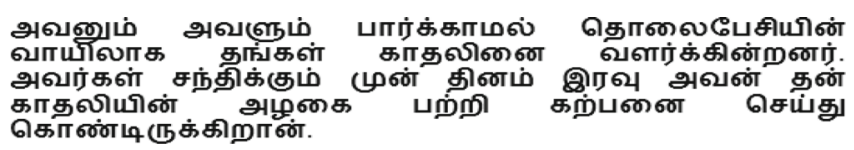

Avanum avalum paarkaamal tholaipesiyin

Vaayilaaga thangal kaadhalinai valarkindranar.

Avargal sandhikkum mun thinam iravu avan than

Kaadhaliyin azhagai pattri karpanai seidhu

konduirukkiraan

Figure 5. Scenario given as input. 
பார்க்காமல் தொலைபேசி காதல் வளர் சந்தி முன் தினம் இரவு

காதலி அழகு கற்பனை செய் கொண்டிரு

Paarkaamal tholaipesi kaadhal valar sandhi mun dhinam iravu

Kaadhali azhagu karpanai sei kondiru

Figure 6. Output of scrutinizer - I.

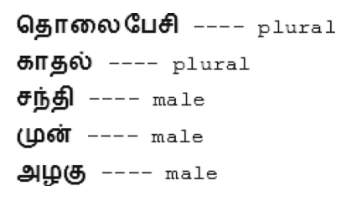

$$
\begin{aligned}
& \text { Tholaipesi ---- plural } \\
& \text { Kaadhal ---- plural } \\
& \text { Sandhi ---- male } \\
& \text { Mun ---- male } \\
& \text { Azhagu ---- male }
\end{aligned}
$$

Figure 7. Output of scrutinizer - II.

have any gender related information in it, and hence, the succeeding words are analysed to get the gender for this word. 'Seidhu kondirukkiraan' has gender information in its suffix, which is 'male' and the same is assigned to the word 'Azhagu'.

4.1c Epitome of the scene: The epitome of the input scene was identified by the ontological interpreter which is shown along with its transliteration in figure 8 . These are the words that are found both in the ontology and the scenario given as input, and hence, this is extracted as the epitome of the scene.

4.1d Adjacency matrix of sub-graph: A sub-graph corresponding to the epitome is extracted from the entire ontology. The adjacency matrix of the sub-graph is shown in figure 9.

The transliteration of the words in the matrix of figure 9 corresponds to the transliteration as given in figure 8.

4.1e Determine longest path: The longest path determined from the sub-graph is shown in figure 10. The links here represent the edges in the ontology and these links have tri-grams in

$\begin{array}{ll}\text { தொலைபேசி } & \text { Tholaipesi } \\ \text { காதல் } & \text { Kaadhal } \\ \text { சந்தி } & \text { Sandhi } \\ \text { முன் } & \text { Mun } \\ \text { காதலி } & \text { Kaadhali } \\ \text { அழகு } & \text { Azhagu } \\ \text { கற்பனை } & \text { Karpanai }\end{array}$

Figure 8. Epitome extracted from the scene. 


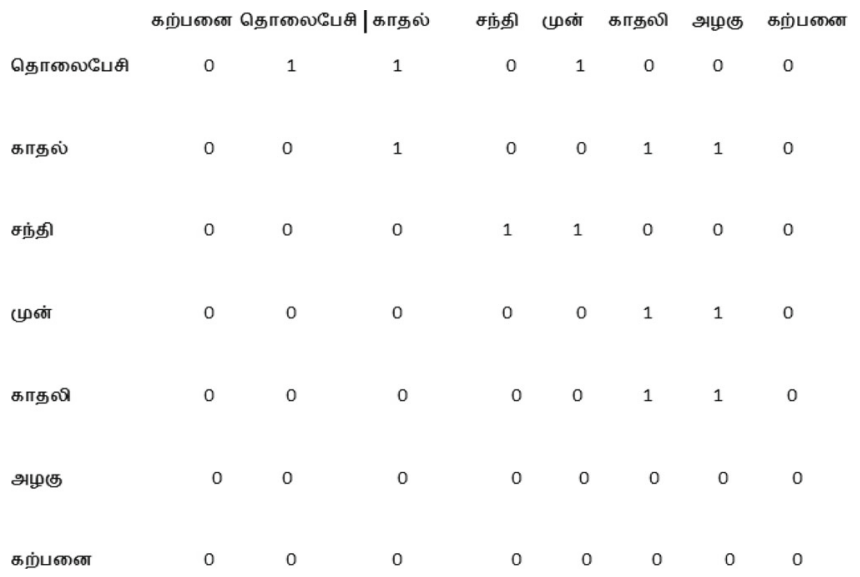

Figure 9. Adjacency matrix of sub-graph.

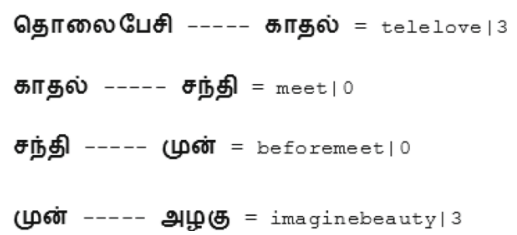

Figure 10. Longest path determined from the sub-graph.

them. Here the link between the nodes 'Tholaipesi' and 'Kaadhal' is represented by a tri-gram 'telelove', and this tri-gram has words that will emphasize on loving without seeing each other. The ' 3 ' in the link indicates how significant this particular link is, in bringing out the semantics in the final lyrics. We have designed to generate $2 x$ lines if ' $x$ ' is the edge weight. Therefore, the edge weight of 3 would result in 6 lines of lyrics getting generated from this particular tri-gram.

4.1f Words received by the morphological generator: The root words are collected from the $N$-gram models by the lyric generator.

These words shown in figure 11 are received by the morphological generator. The first six lines are chosen from the tri-gram 'telelove' and the next six are chosen from the tri-gram 'imagine beauty'. These verses are also transliterated and given in figure 11. As can be seen from the figure 11 the root words that are extracted from the tri-gram are present in their raw form without any suffix information.

4.1g Output lyrics: The lyrics generated by the system for the given scenario are displayed in figure 12.

The lyric is obtained by sending the root words obtained from the tri-grams, which were shown in figure 11 to the morphological generator, thereby coining them to their packed forms 


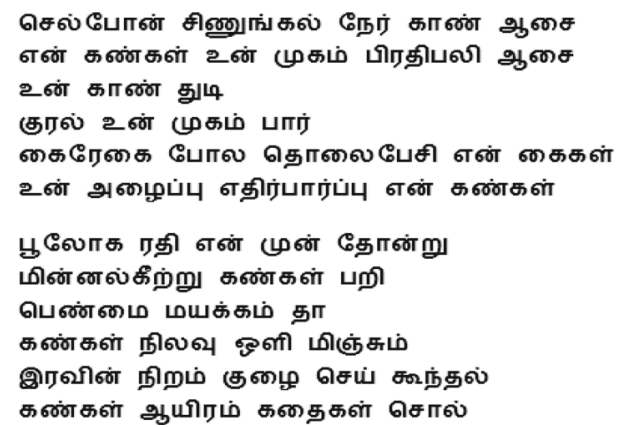

Cellphone sinungal ner kaana aasai

En kangal un mugam pirathipali aasai

Un kaan thudi

Kural un mugam paar

Kairegai pola tholaipesi en kaigal

Un azhaippu ethirpaarppu en kangal

Boologa rathi en mun thondru

Minnalkeettru kangal pari

Penmai mayakkam thaa

Kangal nilavu oli minjum

Iravin niram kuzhai sei koondhal

Kangal aayiram kadhaigal sol

Figure 11. Words received by the morphological generator.

and generating the final lyrics. The final lyrics also have undergone vocative words addition, by looking at the gender information. The transliterated verse is also represented in figure 12. As can be seen from the generated lyrics (refer transliteration) the suffix is added to the verb and noun of the words that were indicated in figure 11. In paragraph 2 of the generated lyrics, the vocative word, 'Sagiye' is added which is a word used to refer to a 'female friend'.

\subsection{Screen shots obtained during testing}

4.2a Sample: The output for a scene which conveys a nostalgic feeling is shown along with its transliteration in figure 13. Here, we have marked the singers by taking the gender information from the scrutinizer. The vocative words are also highlighted in the output shown in figure 13.

\subsection{Performance measures}

In order to measure the effectiveness of the system, we have identified certain performance parameters pertaining to the system, which include the following. The performance analysis was done by conducting a survey with 200 people, including the students, and the teaching and 


\author{
கெல்போண் சிணூ|ங்கல்களை நேரில் காண ஆசை \\ என் கண்களளில் உன் முகும் பிரதிபலிக்க, ஆசை \\ Cellphone sinungalgalai neril kaana aasai \\ En kangalil un mugam pirathipalikka aasai \\ Unnai kaana thudikkiren \\ Kuralil un mugam paarkkiren \\ Kairegai pola tholaipesi en kaigallil \\ Un azhaippin ethirpaarppu en kangalil
}

Sagiye, Boologa rathiyai en mun thondruvayo

Minnalkeettraai kangalai parippayo

Penmai mayakkam tharumaa

Kangal nilavin oliyai minjumaa

Iravin niram kuzhai sei koondhalo

Kangal aayiram kadhaigal sollumo

Figure 12. Output lyrics.

non-teaching staff of the Department of Computer Science and Engineering, Anna University, Chennai. For the performance analysis we took a set of 60 scenes from 100 scenes and tested each scene 5 times. As we chose a random root word to start generating the lyrics, during each trial we arrived at different sets of lyrics, but all conveyed the meaning of the input scenario. Hence, over these 300 trials we showed the output to them and they were asked to score the lyrics with respect to the various parameters. The analysis of the performance of the system with respect to the parameters is discussed below.

4.3a Efficacy measure - Assortment of words $(A W)$ : The effectiveness of the lyrics generated, is typically based on the word set available. Vocabulary is restricted and the knowledge base is not as wide as the language itself. The wideness of the words collection is a crucial parameter. We have around 10,000 words in the corpus, and the average assortment of words in the lyrics generated is calculated as $86.1 \%$. This was done by manually giving a score to each line in the generated lyrics, based on whether the line generated is new or has occurred already. 


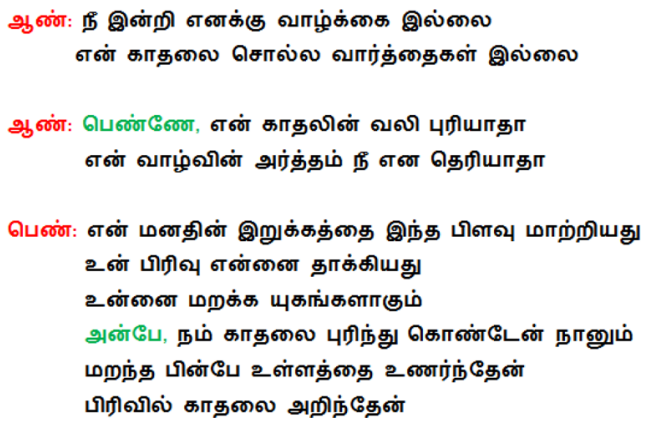

Aan (Male) : nee indri enakku vaazhkai illai

En kaadhalai solla vaarthaigal illai

Aan (Male): Penne, en kaadhalin vali puriyaathaa

En vaazhvin artham nee ena theriyathaa

Pen (Female): En manadhin irukkaththai indha pilavu maatriyathu

Un pirivu ennai thaakkiyathu

Unnai marakka yugangalaagum

Anbe, nam kaadhalai purinthu kondein naanum

Marandha pinbe ullaththai unarnthen

Pirivil kaadhalai arindhen

Figure 13. Another sample output.

Average Assortment of words (AAW) is calculated using the formula

$$
\frac{\sum_{i=1}^{60} \sum_{j=1}^{5} \sum_{k=1}^{n_{i}} a_{i j k}}{\sum_{i=1}^{60} 5 \times n_{i}} \times 100 \%,
$$

where, $i$-scene number, $j$ - trial number, $k$ - line number, $n_{i}-$ the number of lines in the $i^{\text {th }}$ output, $a_{i j k}-1$ if the $k^{\text {th }}$ line is new, 0 if the $k^{\text {th }}$ line is repeated.

4.3b Meter analysis: The meter analysis for the lyrics is based on the number of lines per song. There is no specific deliberation regarding this issue and the system generates the pallavi, anupallavi and charanam, which indicates the three section of the lyrics and this would approximately come to around 10-15 lines.

4.3c Semantic relatedness (SR): This assessment is to find out how well the lyrics generated are in par with the input scene given. The compliance to the given scenario is ensured by an ontological interpretation, which tags the important words that convey the situation in which the song is sung. Also the emotion conveying words is given significance, which guarantees the semantic correctness. We have defined a parameter called 'Average Semantic Relatedness' 
which was calculated by manually giving a score to each line in the generated lyrics, based on how well they are related to the input, and it was found to be $77.3 \%$.

Average Semantic Relatedness (ASR) is calculated using the relationship given by

$$
\frac{\sum_{i=1}^{60} \sum_{j=1}^{5} r_{i j}}{\sum_{i=1}^{60} n_{i}} \times 100 \%,
$$

where, $n_{i}$ - the number of lines in the $i^{\text {th }}$ output, $r_{i j}-1$ if specific, if generic.

The semantic assessment is directly related to the emotion that was identified by the system. The system was also analysed by identifying the emotion without modifying the morphological analyzer. In our input scene, we had nearly $30 \%$ of the scenes that conveyed the emotion based on negation. Hence, the semantic relatedness of those lyrics decreased to $55.2 \%$, as compared to $77.3 \%$ for the other $70 \%$ of the scenes. $55.2 \%$ score was achieved, as we have given 0.5 for the value of $\mathrm{r}_{i j}$ as against 0 .

4.3d Musical score: One of the important parameters for lyrics is how musical it is. This is achieved by ensuring that we have rhyming components, like edhugai, monai, eyaibu used in the system. The system is designed such that the adjacent lines always have the same rhyme.

4.3e Words per line: There are no proper rules on the number of words per line used in poetry, and it is based on the sentence pattern chosen and hence, it is inter-dependent on the various other parameters of poetry. The manual analysis of the outputs of the various scenes shows that the number of words in a line varies from 3 to 8 , based on the sentence pattern.

\subsection{Evaluation of the system in 3 developmental stages}

The system was developed in 3 stages. At the end of each stage the results were analysed to produce a better performance from the system. The systems developmental stages include the following.

4.4a Emotion interpretation with 2000 words: This was the initial stage of the project, wherein the system was first developed to identify just the emotion conveyed in the given scenario, and generate the lyrics for the emotion identified. At this stage, it was found that the lyrics pertained to the emotion, but the semantic details were not exactly portrayed. To elaborate, if the emotion identified was sad, the system generated lyrics that conveyed a sad mood but the reason behind that sadness was not taken into account when generating lyrics, and this makes the results generic.

4.4b Ontological interpretation with 2000 words: Upon studying the results of the previous stage, we understood the need for the intricate semantic details conveyed in the scene, in the process of lyric generation. Hence, we used an ontological approach to learn the concepts conveyed in the scene at a finer level. This gave better results, when the system generated lyrics that did not just convey the emotion, but also portrayed the entire scenario. The lyrics were thus narrative and picturesque. 
4.4c Ontological interpretation with 10,000 words: The ontological approach proved to be robust, but was initially tested with a limited word set of 2000 words.

To study the results with an increased vocabulary, we enhanced the corpus to contain 10,000 words. The semantic details were conveyed in the lyrics but in rare cases, the lines generated were faintly generic. The results achieved for each input are shown in table 1 , and the consolidated results are given in table 2 .

In table 2 , case 1 , we see that the semantic relatedness is very low, because it was done using only the emotional interpretation, and hence, the meaning of the input scenario was not conveyed. In case 2, when the ontological interpretation was adopted, the semantic relatedness increased as we had ensured that the adjacent lines are related, by referring to the input scenario. 2000 words are not sufficient to generate lyrics for the love domain, and hence, we increased the word corpus to 10,000 . But, as can be seen from table 2, case 3, the semantic relatedness reduced;

Table 1. Semantic relatedness and assortment of words for the 60 scenes after including ontological interpretation.

\begin{tabular}{|c|c|c|c|c|c|}
\hline Scene No & SR & AW & Scene No & SR & AW \\
\hline 1 & 56.3 & 79.2 & 31 & 98 & 91 \\
\hline 2 & 93.7 & 92.1 & 32 & 65.5 & 79.2 \\
\hline 3 & 67.4 & 100 & 33 & 77.4 & 68.1 \\
\hline 4 & 71.9 & 62.9 & 34 & 82 & 89.9 \\
\hline 5 & 89 & 92.3 & 35 & 99.7 & 95 \\
\hline 6 & 100 & 86.4 & 36 & 75.3 & 99.7 \\
\hline 7 & 95.3 & 89 & 37 & 82.1 & 84.2 \\
\hline 8 & 57.1 & 53.2 & 38 & 79 & 75 \\
\hline 9 & 68.4 & 90.9 & 39 & 64 & 87.8 \\
\hline 10 & 79 & 67.7 & 40 & 98.1 & 99 \\
\hline 11 & 82.9 & 91 & 41 & 95.2 & 91.2 \\
\hline 12 & 52.4 & 85.5 & 42 & 53.9 & 100 \\
\hline 13 & 81.2 & 97.2 & 43 & 68.4 & 92 \\
\hline 14 & 64.4 & 94.3 & 44 & 73.4 & 63.7 \\
\hline 15 & 99.9 & 72.3 & 45 & 89.2 & 97.3 \\
\hline 16 & 42.7 & 80 & 46 & 58 & 82.1 \\
\hline 17 & 79.1 & 100 & 47 & 99.8 & 100 \\
\hline 18 & 52.6 & 97.8 & 48 & 90 & 58.9 \\
\hline 19 & 89 & 84.2 & 49 & 84.2 & 79.4 \\
\hline 20 & 94.9 & 92.9 & 50 & 79.4 & 85.4 \\
\hline 21 & 74.13 & 93.3 & 51 & 45.6 & 100 \\
\hline 22 & 86.4 & 63 & 52 & 49.7 & 67.7 \\
\hline 23 & 59.7 & 81.1 & 53 & 85 & 89.9 \\
\hline 24 & 81.8 & 90.3 & 54 & 100 & 99.9 \\
\hline 25 & 69 & 99 & 55 & 87.6 & 90 \\
\hline 26 & 97.7 & 92.8 & 56 & 60.3 & 84.4 \\
\hline 27 & 100 & 82.4 & 57 & 78.5 & 93 \\
\hline 28 & 53.5 & 83.9 & 58 & 86.6 & 78.9 \\
\hline 29 & 63.9 & 72.1 & 59 & 55.8 & 100 \\
\hline 30 & 92.3 & 88.9 & 60 & 83.5 & 89.9 \\
\hline
\end{tabular}


Table 2. Average of SR and AW for the three strategies adopted.

\begin{tabular}{llcc}
\hline S.No & \multicolumn{1}{c}{ Strategies adopted } & ASR (\%) & AAW (\%) \\
\hline 1 & Emotion interpretation with 2000 words & 43 & 47 \\
2 & Ontological interpretation with 2000 words & 90.7 & 44.3 \\
3 & Ontological interpretation with 10,000 words & 77.3 & 86.1 \\
\hline
\end{tabular}

however, the assortment of words increased. The increase in assortment of words is due to the availability of large data, and the random choice of words. But the semantic relatedness reduced, which is due to the randomness included in the system. For example, during the lyric generation of even lines, our algorithm starts from the ending word by determining a rhyming word that matches the previous line. In this case, there will be many rhyming words. Randomly, any one of them would be chosen. This choice reduced the semantic coherence of the lines. However, the algorithm still retained the semantic relatedness of the lyrics in the odd lines, which is the reason for achieving $77.3 \%$ of ASR measure. We tried increasing the corpus by 200 more words, and still achieved nearly $77 \%$ of the ASR measure for the same set of scenes.

The results of tables 1 and 2 are pictorially represented in the figures below. The sectors in figure 14 show the proportions of the semantic relatedness (SR) obtained for the various inputs, and most inputs are found to lie in the SR range of 80-90\%. The sectors in figure 15 show the proportions of the assortment of words (AW) obtained for the various inputs and most inputs are found to lie in the AW range of $90-100 \%$.

\subsection{Turing test}

A Turing test is one, in which a human interrogator is isolated, and given the task of distinguishing between a human and a computer, based on their replies to questions that the interrogator poses. After a series of tests are performed, the interrogator attempts to determine, which subject

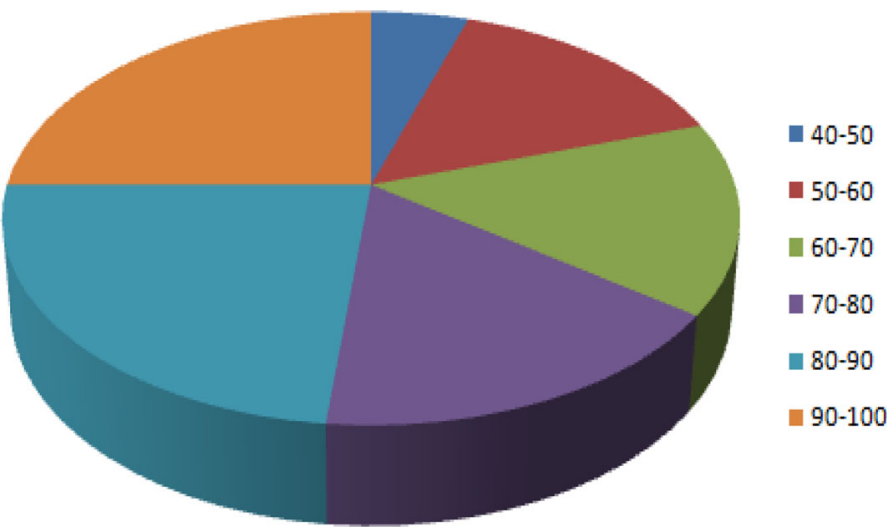

Figure 14. SR values obtained for the 300 trials. 


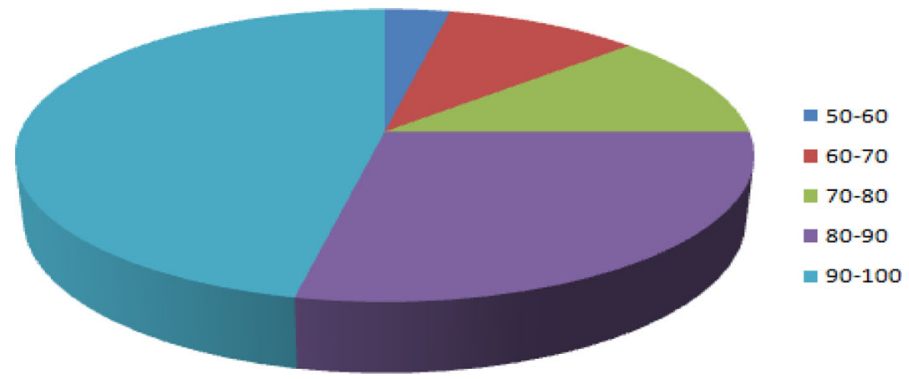

Figure 15. AW values obtained for the 300 trials.

Table 3. Results of Turing test.

\begin{tabular}{lcc}
\hline Variety of input & Interpreted as human (\%) & Interpreted as machine (\%) \\
\hline Human generated & 70 & 30 \\
Machine generated & 64.6 & 35.4 \\
\hline
\end{tabular}

is human, and which is an artificial intelligence machine. The computer's success at thinking can be quantified by its probability of being misidentified as a human subject. This kind of a test was performed with the results obtained from the lyric generation system. The lyrics obtained from the system were mixed with the human generated ones and given to the end users. We took 10 output samples from our system, along with 10 other human written lyrics for this test. The lyrics that were generated by human are taken from Tamil film songs which were written by Tamil film lyricists. These human written lyrics are not popular. The reason for not selecting popular songs is to prevent the survey from being biased as the audience would be aware of the lyrics in a popular song. We solicited nearly 300 end users via Facebook (Sridhar et al 2012a) who were naive to this system to rate the lyrics. The end users were asked to rate the lyrics through a poll, and to identify if they were human or machine generated. The statistical report of the Turing test is tabulated below in table 3 .

\subsection{Feedback from end users}

In addition to the Turing test, the end users were also asked to cast their votes via a poll, to learn how the lines in the lyrics have attracted them, and their comments on the overall validity of the system. The votes given by the end users, and the comments received are portrayed in a bar chart, shown in figures 16 and 17.

Figure 16 shows the comments received for the machine generated lyrics, and figure 17 shows the comments received for human generated lyrics. Thus, we can conclude that our system has generated lyrics, which are as good as human generated lyrics.

The system has attracted more than 300 users in 3 days. The users of varying age groups have responded to the polls of the page. The statistics of the same are shown here in figure 18. 

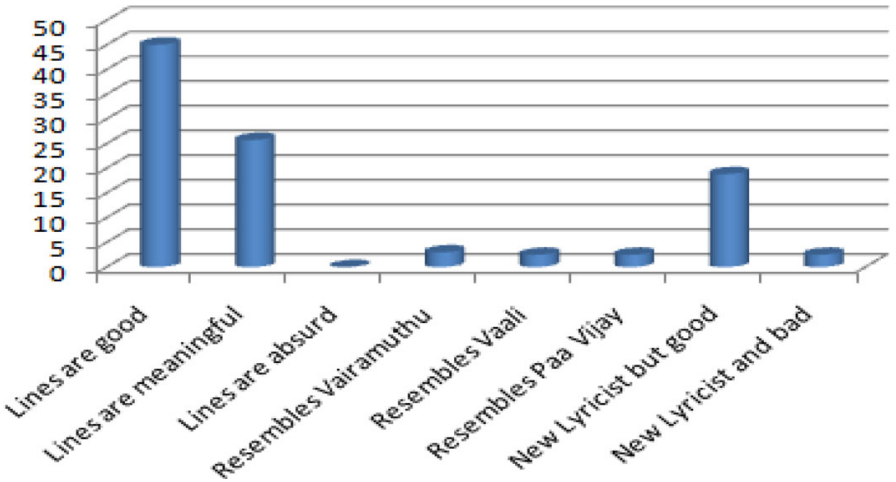

Figure 16. Bar chart built for comments obtained for machine generated lyrics.

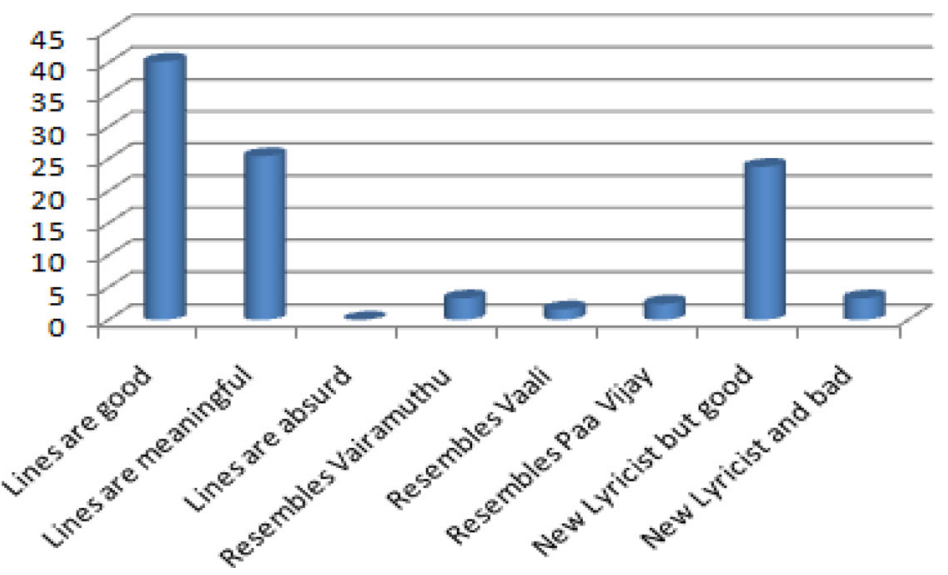

Figure 17. Bar chart built for comments obtained for human generated lyrics.

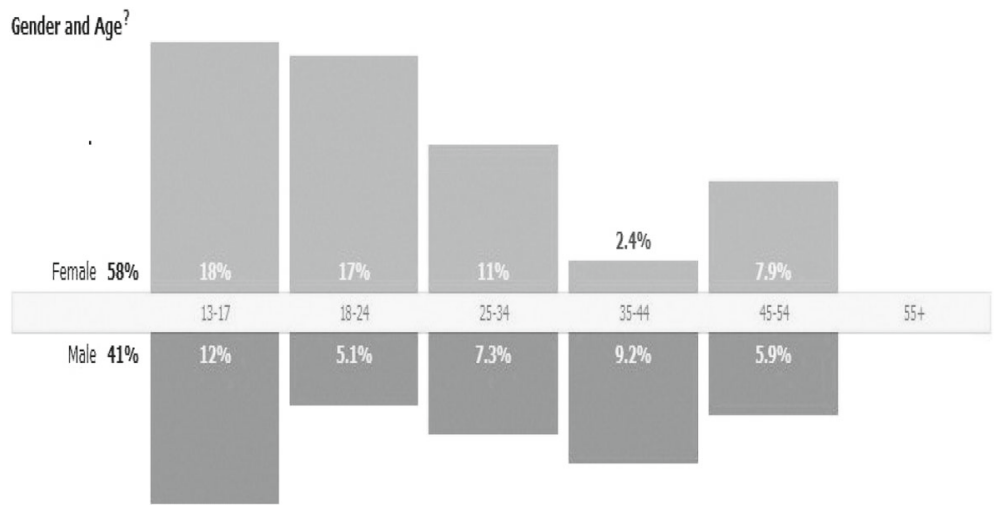

Figure 18. Graph obtained from Facebook analysis section of our 'lyric generation' page. 


\section{Conclusion and future work}

This work on lyric generation is based on analysing the input situation which is available in a text and processing it. On the basis of these studies it is concluded that the system architecture is very versatile, and helps in identifying the semantics to convey the concepts in the input scenario. With a well-defined ontology, the system is able to understand the scene clearly. Thus, the finer and intricate details of the scenario are evidently observed and the system is able to generate lyrics similar to those written by human lyricists. With a rich collection of words, the lyrics generated are highly narrative and they portray the scene in an effective manner. As ontology is the heart of the system, it must be very extensive and enhanced. A domain independent ontology must be constructed to handle any given scenario to generate lyrics for the same. Once this is done, it will be possible to predict the scenario, from the generated lyrics.

The poetry clauses of Tamil language like venpaa, aasiriyappa, kalippa, and vanjippaa are not considered. This work can be extended to generate lyrics that would belong to one of these poetry clauses. Timeline-based lyric generation is not targeted. When a scenario that starts with a complete mood of happiness and ends in total sadness is given as input, in the resulting lyrics also there will be sudden transition in the mood, which is a bit contradictory in certain cases. Some strategies could be tried in future to eliminate this effect. In addition, our generated lyrics are based on the input text. The choice of words is done at random, and hence, the generated lyrics may not be singable. Hence, we could consider 'tune' as an additional parameter and modify the words in the generated lyrics, so that they become singable. Example-based lyric generation could be attempted, which we have not tackled here. The use of geographic information could also be attempted, for generating lyrics.

\section{Acknowledgements}

We thank the Coordinator, Tamil Computing Lab, Department of Computer Science and Engineering (DCSE), Anna University, Chennai for providing the morphological analyzer module.

\section{References}

Ananth Ramakrishnan A, Kuppan S and Sobha L D 2009 Automatic generation of Tamil lyrics for melodies. In: Proc. NAACL HLT Workshop on Computational Approaches to Linguistic Creativity (CALC), pp. 40-46

Apte C and Weiss S 1997 Data Mining with Decision Trees and Decision Rules. In: Future generation computer systems, vol. 13, No. 2-3, pp. 197-210

Bowra C M 1961 Greek Lyric Poetry - From Alcman to Simonides, Oxford University Press

Hirschman L and Gaizauskas R 2002 Natural language question answering: the view from here. J. Natural Language Eng. 7(4): 275-300

Karthika R, Geetha T V, Ranjani P and Karky M 2011 Lyric Mining: Word, Rhyme \& Concept Co-occurrence Analysis. In: Proc. Internat. Tamil Internet Conf., 2011, pp. 276-281

Mahalingam K and Huhns M N 1998 Representing and Using Ontologies, Center for Information Technology, Thesis, University of South Carolina

Mann C W and Moore A J 1981 Computer Generation of Multiparagraph English text. American J. Comput. Linguist. 7(1): 17-29

Oliveira H G 2009 Portugal PoeTryMe: Towards Meaningful Poetry Generation, Dissertation, Universidade de Coimbra Portugal 
Pandian L S and Geetha T V 2009 Semantic Role based Tamil Sentence Generator. In: Internat. Conf. on Asian Languages Processing, pp. 80-85

Sobha L D and Ananth Ramakrishnan A 2010 An alternate approach to meaningful lyric generation in Tamil. In: Proc. NAACL HLT 2010 Second Workshop on computational Approaches to Linguistic Creativity, pp. 31-39

Spyns P, Meersman R and Jarrar M 2002 Data modelling versus ontological engineering. Special Issue of SIGMOD Record on Semantic Web, Database Management and Information Systems 31(4): 12-17

Sridhar R et al 2012a Lyric Generation, http://www.facebook.com/LyricGeneration

Sridhar R, Jalin Gladis D, Ganga K and Dhivya Prabha G 2012b N-gram based approach to automatic Tamil lyric generation by identifying emotion. In: Proceedings of International Conference on Advances in Computing, 2012, pp. 919-926

Suriyah M, Karky M, Geetha T V and Parthasarathi R 2011 Special Indices for LaaLaLaa Lyric Analysis \& Generation Framework. In: Proc. Internat. Tamil Internet Conf., pp. 287-292

Thomas C H, Leiserson C E, Rivest R L and Stein C 2008 Introduction to algorithms, Prentice Hall of India Weiss M A 2006 Data Structures and algorithms analysis in C, Pearson Education, India

Wong T M 2008 Automatic Generation of Modern Haiku Poetry, Dissertation, City University of Hong Kong 